\title{
A NOTE ON EVERYWHERE DENSE SUBGROUPS
}

\section{B. J. PETTIS ${ }^{1}$}

In the first issue of these Proceedings [1] ${ }^{2}$ Hsien-Chung Wang has shown that in any separable nondiscrete locally compact and metric group there is always an uncountable everywhere dense proper subgroup. Since any countable subset of a nondiscrete $T_{1^{-}}$ space is first category, this result is implied by each of the following conjectures, in which $X$ is an arbitrary topological group and $G$ any proper subgroup of $X:\left(\mathrm{C}_{1}\right)$ if $X$ is uncountable, $G$ lies in some uncountable proper subgroup; $\left(\mathrm{C}_{2}\right)$ if $X$ is second category, $G$ lies in some second category proper subgroup. Using Wang's methods we here verify these conjectures for certain special cases and also establish his theorem with "locally compact and metric" replaced by "second category and Hausdorff."

Let $X$ be any group with $e$ the identity element and let $\Phi$ be a family of functions on $X$ to $X$ including the identity function $\Phi(x)=x$. Let \& denote the class of all non-null proper $\Phi$-subgroups $G$ in $X$, " $\Phi$-subgroup" meaning a subgroup $G$ such that $\phi(G) \subset G$ for every $\phi \in \Phi$. For each $G \in \circlearrowleft)$ take $Y_{1}(G)$ to consist of all pairs $(1 ; g)$ with $g \in G$, and for $n \geqq 2$ let $Y_{n}(G)$ be composed of all $2 n$-tuples $y$ of the form $y=\left(n ; g_{1}, \cdots, g_{n} ; \phi_{1}, \cdots, \phi_{n-1}\right)$ with $g_{i} \in G$ and $\phi_{i} \in \Phi$. Set $Y_{G}=\cup_{1}^{\infty} Y_{n}(G)$ and let $\psi_{G}$ on $X \times Y_{G}$ to $X$ be defined by $\psi_{G}(x, y)$ $=g$ when $y=(1 ; g)$ and $\psi_{G}(x, y)=g_{1} \phi_{1}(x) g_{2} \phi_{2}(x) \cdots g_{n-1} \phi_{n-1}(x) g_{n}$ when $y \in Y_{n}(G)$ for $n \geqq 2$. For fixed $G$ in BS and $x$ in $X$ we write $G(x)$ for the set $\psi_{G}\left(x ; Y_{G}\right)$ and note that $G(x)$ is always a semigroup in $X$ and that $G(x) \ni x$ since $Y_{G} \ni(2 ; e, e ; \bar{\phi})$. Moreover, $G(x) \supset \psi_{G}\left(x, Y_{1}(G)\right)$ $=G$; hence $G(g)=G$ when $g \in G$, since $\phi_{i}(g) \in G$ for $g \in G$ and $\phi_{i} \in \Phi$. For each $q \in X$ set $S_{G}(q)=[x \mid G(x) \ni q]$ and define $S_{G}(Q)$ for any set $Q$ in $X$ to be $U\left[S_{G}(q) \mid q \in Q\right]$. Finally, for any fixed element $G_{0}$ of $B$ and any non-null subset $P$ of the complement $X-G_{0}$ of $G_{0}$, let $\$\left(G_{0}, P\right)$ be all $G$ in $\& 5$ which contain $G_{0}$ and are disjoint to $P$. We note that by an obvious application of the Hausdorff Maximality Principle $\mathbb{B}\left(G_{0}, P\right)$ always contains at least one maximal element.

THEOREM 1. Let $G_{0} \in \circlearrowleft$ and $P \subset X-G_{0}$ be fixed. Suppose that $\mathfrak{M}$ is a non-null class of maximal elements of $\leftrightarrow\left(G_{0}, P\right)$ such that (i) $M(x)$ is a

Presented to the Society April 21, 1951; received by the editors February 2, 1951 and, in revised form, July 14, 1951.

1 This paper was written under Contract N7-onr-434, Task Order III, Navy Department (The Office of Naval Research).

2 Numbers in brackets refer to the references at the end of the paper. 
$\Phi$-subgroup for every $M \in \mathbb{M}$ and every $x \in X$. Then $S_{M}(P)=X-M$ for each $M \in \mathfrak{M}$. If $P$ is countable and $₹$ is a class of subsets of $X$ such that (ii) \& is closed under countable unions and (iii) $S_{M}(p) \in \mathbb{E}$ for every $M \in \mathfrak{M}$ and every $p \in P$, it follows that $X-M \in \mathbb{f}$ for each $M \in \mathfrak{M}$, and hence $X \in \mathbb{F}$ if $\mathfrak{M}$ contains an element of $\mathfrak{F}$.

Fix $M$ in $\mathfrak{M}$ and consider any $q$ not in $M$. From (i) we know that $M(q)$ is a $\Phi$-subgroup and, as noted above, that $M(q) \supset M \supset G_{0}$ and $M(q) \ni q$. From the maximality of $M$ in $\$\left(G_{0}, P\right), M(q)$ is not in $\circlearrowleft\left(G_{0}, P\right)$ and hence $M(q) \ni p_{0}$ for some $p_{0}$ in $P$. Thus $q \in S_{M}\left(p_{0}\right)$ $\subset S_{M}(P)$, so that $M \cup S_{M}(P)=X$. On the other hand, $M$ and $S_{M}(P)$ are disjoint; for if $m \in M$, then $M(m)=M$, while if $m \in S_{M}(P)$, then $M(m)$ and $P$ intersect; since $M$ and $P$ are disjoint, so are $M$ and $S_{M}(P)$. Thus $X-M=S_{M}(P)$. If $P$ is countable and (ii) and (iii) hold, obviously $S_{M}(P)=\mathrm{U}_{p} S_{M}(p)$ must be an element of $\&$, so that $X-M \in \mathbb{E}$.

In the next two theorems $X$ is a topological group, $\sqrt{ }$ is the class of countable subsets of $X$, and $₹$ consists of the first category sets. The following condition on $\Phi$ will be considered: $(0.1) G(x)$ is a $\Phi$-subgroup for each $G$ in (S) and each $x$ in $X$.

THEOREM 2. Suppose that $X$ is second category nondiscrete and Hausdorf, that $\Phi$ is countable and satisfies (0.1), and that each $\phi$ is continuous. If $G_{0} \in(5)$ is everywhere dense in $X$, then $G_{0} \subset M$ for some element $M$ of $(5)$ that is uncountable and everywhere dense in $X$.

Choose any countable non-null subset $P$ of $X-G_{0}$ and let $M$ be a fixed maximal element of $\$\left(G_{0}, P\right)$. It suffices to show that $M$ is uncountable. To do this we assume that $M \subset \mathcal{E}$ and arrive at a contradiction via Theorem 1 . Let $\mathfrak{M}=\{M\}$. We first note that $M$ is in both $\mathfrak{M}$ and $\mathbb{E}$, since $\mathbb{E} \supset \mathfrak{S}$ due to $X$ being a nondiscrete $T_{1}$-space; if the hypotheses of Theorem 1 are verified, it will then follow that $X$ is in (E, contrary to $X$ being second category. But (i) of Theorem 1 results from (0.1), and (ii) is obvious. Concerning (iii) we observe that $Y_{M}$ is countable since $M$ and $\Phi$ are, and hence for any $p$ in $P$ the set $S_{M}(p)$ is the countable union of sets $S_{M}(p, y) \equiv\left[x \mid \psi_{M}(x, y)=p\right]$. Since $X$ is a $T_{1}$-space and $\psi_{M}$ is continuous in $x$ for fixed $y$ (each $\phi$ being continuous), each $S_{M}(p, y)$ is closed in $X$. By the first part of Theorem 1 we have $S_{M}(p, y) \subset S_{M}(P) \subset X-G_{0}$; hence $S_{M}(p, y)$, being closed and in the complement of everywhere dense $G_{0}$, must be nowhere dense. This clearly implies that $S_{M}(p) \in \mathbb{E}$ for every $p$ in $P$, ending the proof.

The next theorem involves this condition on $\Phi:(0.2)$ for each choice of $k$, of $\phi_{1}, \cdots, \phi_{k}$ in $\Phi$, and of $Q \in \mathcal{E}$ with $e \notin Q$, the set 
$\left[x \mid \phi_{1}(x) \cdots \phi_{k}(x) \in Q\right]$ is in $\mathbb{E}$.

Theorem 3. Suppose that $X$ is second category and Abelian, and that $\Phi$ is countable and satisfies (0.1) and (0.2). Then for any $G_{0}$ in (B) there is some second category element $M$ of \& such that $M \supset G_{0}$.

As in the proof of Theorem 2 we choose $P$ to be any countable non-null subset of $X-G_{0}, M$ to be any maximal element of $\&\left(G_{0}, P\right)$, and $\mathfrak{M}$ to be $\{M\}$; this time we have only to prove that $M$ is second category. Assuming the contrary, we have $M$ in both $\mathfrak{M}$ and $\mathfrak{E}$, and if the hypotheses of Theorem 1 are fulfilled, we can conclude that $X \in \mathbb{E}$, contrary to assumption. Since (0.1) holds for $\Phi$, we again have (i) of Theorem 1 satisfied. To verify (iii) we observe that since $X$ is Abelian and $M \cap P$ is null, we can, for each $p \in P$, write $S_{M}(p)$ $=\left[x \mid m \phi_{1}(x) \cdots \phi_{k}(x)=p\right.$ for some $m \in M$ and some $\phi_{1}, \cdots, \phi_{k}$ in $\Phi$ with $k \geqq 1]$. Thus $S_{M}(p)$ is the union of sets $\left[x \mid \phi_{1}(x) \cdots \phi_{k}(x)\right.$ $\left.\in M^{-1} p\right]$ for varying $k$ and varying $\phi_{i}$ in $\Phi$. Since $e \notin M^{-1} p$ and $M^{-1} p$ $\in \Subset$, we can apply $(0.2)$ and the countability of $\Phi$ to conclude that $S_{M}(p) \in \mathbb{E}$ for each $p \in P$, which completes the proof.

As an application of Theorems 2 and 3 let $\Phi$ consist of $\Phi$ and $\phi^{\prime}$ where $\phi^{\prime}(x)=x^{-1}$. Here (B) consists of all proper subgroups of $X$, and $(0.1)$ is satisfied. Any function $\phi_{1}(x) \cdots \phi_{k}(x)$ with $\phi_{i} \in \Phi$ is continuous and has the form $x^{r}$ for some integer $r$; recalling that any continuous function mapping non-null open sets into somewhere dense sets has its inverse function sending first category sets into first category sets [2], it follows that (0.2) is satisfied by this $\Phi$ if $x^{r}$ sends non-null open sets into somewhere dense sets whenever $x^{r}$ is not identically $e$ and $r>0$. We thus have the following theorems.

THEOREM 4. An everywhere dense proper subgroup $G_{0}$ of a second category nondiscrete Hausdorff group $X$ must lie in some uncountable everywhere dense proper subgroup $M$.

Theorem 5. Suppose that $X$ is second category and Abelian and that for each integer $r>0$ such that $x^{r} \neq \equiv$ the map $x^{r}$ sends non-null open sets into somewhere dense sets. Then any proper subgroup of $X$ lies in some second category proper subgroup $M$.

From Theorem 4 we have the following.

THEOREM 6. Let $X$ be a second category nondiscrete Hausdorff group containing a countable everywhere dense subset $D$. Then any proper subgroup $G$ of $X$ lies in an uncountable proper subgroup $M$ of $X$; if $G$ is countable, $M$ can be taken to be everywhere dense as well.

When $G$ is uncountable, take $M=G$. When $G$ is countable, let 
$G_{0}$ be the countable subgroup generated by $G \cup D$; then $G_{0}$ is everywhere dense, and $G_{0} \neq X$ holds since $X$ is uncountable. Theorem 4 now yields the desired $M$.

Taking $G$ to be the trivial subgroup in Theorem 6 provides the previously stated extension of Wang's result.

We note in Theorems 3 and 5 that the second category subgroup $M$ will be uncountable if $X$ is Hausdorff, and that if $X$ is connected, $M$ will be everywhere dense and, by a theorem of Banach [4], also non-Baire. From this and from Theorem 5 and a result of Gleason [3, Theorem 3 and corollary] we have the following theorem.

Theorem 7. When $X$ is Abelian and locally Euclidean, any proper subgroup $G$ of $X$ is contained in some uncountable second category proper subgroup $M$; if $X$ is also connected, $M$ must be everywhere dense and non-Baire.

Theorem 5 and the remark preceding Theorem 7 also show the following: $(\alpha)$ in the algebra of Lebesgue measurable sets in $n$-dimensional space any proper subalgebra is contained in a proper second category everywhere dense non-Baire subalgebra, and $(\beta)$ in any second category real linear topological space any proper subgroup (and hence any proper linear subspace) is contained in a second category everywhere dense non-Baire proper subgroup. We remark also that in $(\beta)$ we can, by Theorem 3, replace "subgroup" by "rational subspace"; thus, for example, any proper additive subgroup of the reals (for example, the rationals) is contained in a subgroup of the same sort that in addition is second category, everywhere dense, non-Baire, and closed under multiplication by rationals.

For another application of Theorems 2 and 3 let $X$ be a ring with unit 1 and let $Z$ be any countable additive subgroup that contains 1 and is also a multiplicative semigroup. Set $\Phi=\left[\phi_{z}\right]$ where $\phi_{z}(x)=z x$ and $z \in Z$. Here $\Phi$ is countable and contains $\Phi$, and (0.1) is satisfied. By the same remark (preceding Theorem 4) that justified Theorem 5 it follows that (0.2) is satisfied if this is true: (0.3) for any fixed $z$ in $Z$ either $z x=0$ for all $x$ or else $z V$ is somewhere dense whenever $V$ is non-null and open. From Theorems 2 and 3 we have the following theorem.

THEOREM 8. Suppose that the additive group of $X$ is a second category topological group and that $z x$ is continuous in $x$ for each fixed $z$ in $Z$. Let $G_{0}$ be a proper additive subgroup with $z G_{0} \subset G_{0}$ for every $z \in Z$. (I) If the additive group of $X$ is a nondiscrete and Hausdorff and $G_{0}$ is everywhere dense, there is an uncountable everywhere dense proper additive subgroup $M$ containing $G_{0}$ and having $z M \subset M$ for each $z \in Z$. (II) 
If $Z$ satisfies (0.3), there is a second category proper additive subgroup $M$ containing $G_{0}$ and with $z M \subset M$ holding for every $z \in Z$.

The following example shows that in the above theorems any lack of assertion that $M$ can be chosen to be everywhere dense is a necessary lack. Let $X$ be the product of the reals with the integers $\bmod 2$, and let $G=[(r, 0) ; r$ real $]$; here $X$ satisfies all the hypotheses in these theorems, and $G$ is a second category (in $X$ ) uncountable maximal proper subgroup that is not everywhere dense.

In conclusion we remark that much of the above can be carried through with groups replaced by semigroups with units, since Theorem 1 holds when this substitution is made.

\section{REFERENCES}

1. H. C. Wang, On a problem of P. A. Smith, Proceedings of the American Mathematical Society vol. 1 (1950) pp. 18-19.

2. A. D. Wallace, Some characterizations of interior transformations, Amer. J. Math. vol. 61 (1939) pp. 757-763.

3. A. M. Gleason, Square roots in locally Euclidean groups, Bull. Amer. Math. Soc. vol. 55 (1949) pp. 446-449.

4. B. J. Pettis, Remarks on a theorem of E. J. McShane, Proceedings of the American Mathematical Society vol. 2 (1951) pp. 166-171.

TULANE UNIVERSITY 